\title{
Discovery of Extended Infrared Emission around the Neutron Star RXJ0806.4-4123* ${ }^{* \dagger}$
}

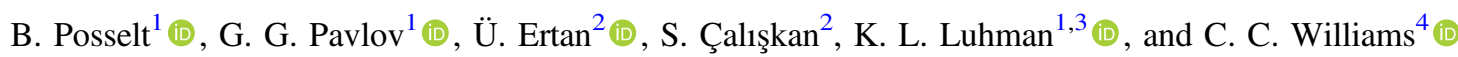 \\ ${ }^{1}$ Department of Astronomy \& Astrophysics, Pennsylvania State University, 525 Davey Lab, University Park, PA 16802, USA; posselt@psu.edu \\ ${ }^{2}$ Sabanc1 University, 34956, Orhanlı Tuzla, İstanbul, Turkey \\ ${ }^{3}$ Center for Exoplanets and Habitable Worlds, The Pennsylvania State University, University Park, PA 16802, USA \\ ${ }^{4}$ Steward Observatory, University of Arizona, 933 North Cherry Avenue, Tucson, AZ 85721, USA \\ Received 2018 January 17; revised 2018 July 6; accepted 2018 July 27; published 2018 September 17
}

\begin{abstract}
Following up on a faint detection of a near-infrared (NIR) source at the position of the X-ray thermal isolated neutron star RX J0806.4-4123, we present new Hubble Space Telescope observations in the H-band. The NIR source is unambiguously detected with a Vega magnitude of $23.7 \pm 0.2$ (flux density of $0.40 \pm 0.06 \mu \mathrm{Jy}$ at $\lambda=1.54 \mu \mathrm{m}$ ). The source position is coincident with the neutron star position, and the implied NIR flux is strongly in excess of what one would expect from an extrapolation of the optical-UV spectrum of RX J0806.4-4123. The NIR source is extended, with a size of at least 0 ." 8 , and shows some asymmetry. The conservative upper limit on the flux contribution of a point source is 50\%. Emission from gas and dust in the ambient diffuse interstellar medium can be excluded as a cause for the extended emission. The source parameters are consistent with an interpretation as either the first NIR-only detected pulsar wind nebula or the first resolved disk around an isolated neutron star.
\end{abstract}

Key words: brown dwarfs - infrared: general - pulsars: individual (RXJ0806.4-4123) - stars: neutron

\section{Introduction}

The X-ray pulsar RX J0806.4-4123 belongs to a group of seven nearby $(<1 \mathrm{kpc})$, X-ray thermal isolated neutron stars (XTINSs, also dubbed the "Magnificent Seven") whose defining properties are as follows: a lack of detected nonthermal X-ray emission, radio-quietness, relatively large pulse periods (3-11 s), and inferred dipole magnetic fields on the order of $\sim 10^{13} \mathrm{G}$. The observational properties of the XTINSs (e.g., Kaplan \& van Kerkwijk 2009; Haberl 2013) place this neutron star population between those of the rotation-powered pulsars and the magnetars. Since their X-ray luminosities exceed their spin-down energies by a factor of at least 10 , explaining the XTINS properties requires an additional energy source other than the energy loss of a rotating dipole. Currently, it is commonly assumed that the most promising model for the heating of magnetars and XTINSs is the decay of their large magnetic fields. Viganò et al. (2013) presented simulations of a magnetothermal evolution model capable of unifying the diverse neutron star populations. In this model, magnetars and XTINSs are hot because they are heated by the decay of their large magnetic fields. In an alternative model, the observational properties of the XTINSs and magnetars are explained by formation of and influence by supernova fallback disks (e.g., Ertan et al. 2014). However, the fallback disk model alone cannot explain the giant outbursts of magnetars. Acceptance of this model is also hampered by the lack of fallback disk detections. So far, there is only one example of a

\footnotetext{
* Based on observations made with the NASA/ESA Hubble Space Telescope, obtained at the Space Telescope Science Institute, which is operated by the Association of Universities for Research in Astronomy, Inc., under NASA contract NAS 5-26555. These observations are associated with program GO14745 .

$\dagger$ Based on observations obtained at the Gemini Observatory, which is operated by the Association of Universities for Research in Astronomy, Inc., under a cooperative agreement with the NSF on behalf of the Gemini partnership: the National Science Foundation (United States), the National Research Council (Canada), CONICYT (Chile), Ministerio de Ciencia, Tecnología e Innovación Productiva (Argentina), and Ministério da Ciência, Tecnologia e Inovação (Brazil).
}

possible (passive) fallback disk around the $3.9 \mathrm{kpc}$ distant anomalous X-ray pulsar 4U 0142+61, detected with Spitzer IRAC by Wang et al. (2006). Ertan et al. (2007) reported that an active irradiated disk model can also reproduce the observed optical and infrared fluxes of $4 \mathrm{U} 0142+61$.

RX J0806.4-4123 has the second-longest period among the XTINSs, $P=11.37 \mathrm{~s}$. Its spin-down power is $\dot{E}=1.6 \times 10^{30}$ $\mathrm{erg} \mathrm{s}^{-1}$, its inferred dipole magnetic field is $B=2.5 \times 10^{13} \mathrm{G}$, and its total X-ray luminosity at a distance of $250 \mathrm{pc}$ is $L_{X}=2.6 \times 10^{31} \mathrm{erg} \mathrm{s}^{-1}$ (Haberl et al. 2004; Haberl 2007; Posselt et al. 2007; Kaplan \& van Kerkwijk 2009). Previously, we used the Very Large Telescope (VLT) in the near-infrared (NIR) to carry out a direct-imaging search for substellar companions around isolated XTINSs (Posselt et al. 2009), and we noticed a slight $H$-band flux enhancement at the location of RX J0806.4-4123. Motivated by an additional Herschel $160 \mu \mathrm{m}$ detection very close to the neutron star position (Posselt et al. 2014), we followed up on the NIR source with the Gemini telescope. We indeed found indications of the VLT source in another NIR band. The two independent faint flux enhancements together, $H_{\mathrm{VLT}} \approx 23.3 \pm 0.5(1 \sigma)$ and $J_{\text {Gemini }} \approx 24.8 \pm 0.5(1 \sigma)$, resulted in a combined NIR detection ( $H$ and $J$ bands) significance of $3.1 \sigma$ (Posselt \& Luhman 2016). From the UV-optical spectral slope (measured by Kaplan et al. 2011), one would expect the XTINS to have a NIR magnitude on the order of 28, much fainter than the NIR detection. The NIR flux enhancements can be interpreted as a substellar companion, a fallback disk, or even a very unusual pulsar wind nebula (PWN). In this paper, we report the results of recently obtained Hubble Space Telescope (HST) NIR imaging follow-up observations that were carried out to confirm the previous weak NIR detection.

\section{Data Reduction and Results}

\subsection{HST Data}

We obtained NIR images (program GO-14745) of RX J0806.4 -4123 with the HST Wide Field Camera 3 (WFC3; Kimble 


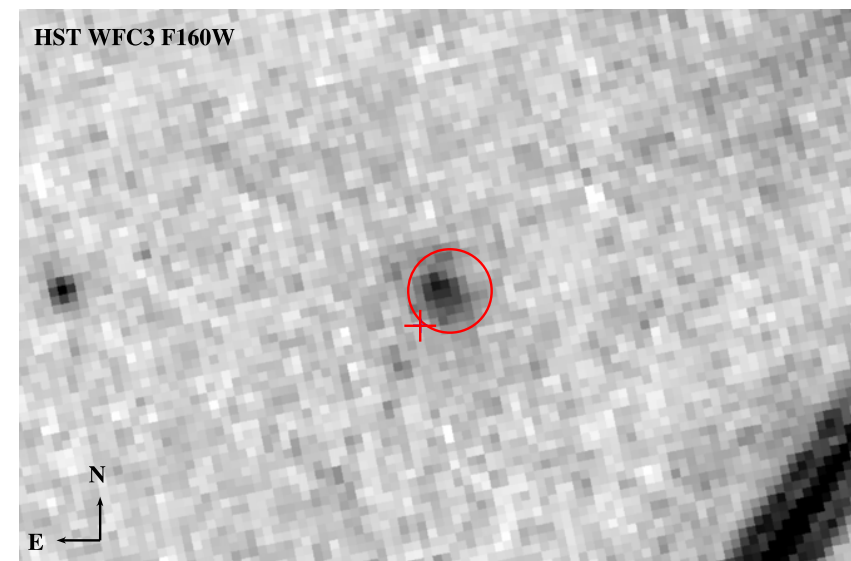

Figure 1. 2016 HST F160W image $\left(6^{\prime \prime} \times 4^{\prime \prime}\right)$ at the position of RX J0806.4-4123. The red circle $(r=0$."3) is centered on the previous (2009 and 2010) HST F475W detection (STmag = 27.9) of the neutron star by Kaplan et al. (2011), and the red cross marks the 2002 Chandra position that has a positional uncertainty of 0 .! 6 (Haberl et al. 2004).

et al. 2008) on 2016 September 11 (MJD 57642.98), employing the F160W filter with a pivot wavelength of $1.537 \mu \mathrm{m}$. The total exposure time was $5417 \mathrm{~s}$. We used the WIDE-6 POS-TARGs dither pattern (optimizes the subsampling of the pixels; HST Instrument Science Report 2016-14 ${ }^{5}$ ) for our 6 individual exposures in MULTIACCUM mode (linear SPARS timing sequence). We stacked and processed our images within PyRAF using AstroDrizzle (version 2.1.8) of the DrizzlePAC software (Fruchter et al. 2010; Gonzaga et al. 2012). Since our target source is very faint, we used the inverse variance map (IVM) weighting scheme for the final combination of the data (Gonzaga et al. 2012). We experimented with the drizzle parameters for a balance of spatial resolution and sampling noise. We chose a final pixel scale of 0. " 0642 pixel $^{-1}$ and a pixel fraction of 0.7 . We registered our astrometry to the Gaia data release 1 (Gaia Collaboration et al. 2016a, 2016b); employing the common 117 reference sources in the field of view, the rms of our astrometry fit is 0 " 019 .

RX J0806.4-4123 was observed in X-rays in 2002, 2005, and 2015 with Chandra. From these observations the proper motion

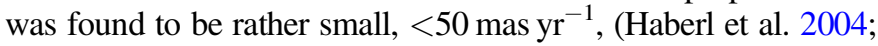
Motch et al. 2009; B. Posselt et al. 2018, in preparation) RX J0806.4-4123 was also observed and detected with HST in the UV (ACS/SBC F140LP; STmag = 23.61 \pm 0.11 ) and in the optical (ACS/WFC F475W, STmag $=27.92 \pm 0.22$ ) in 2009 and 2010 (Kaplan et al. 2011). The neutron star's position in Xrays, in particular in the optical $\mathrm{F} 475 \mathrm{~W}$ band, are consistent with an unambiguous HST detection in the F160W band; see Figure 1.

To measure the source flux we used aperture measurements with a sky annulus for the background and noise estimate. We used the daophot and photcal IRAF packages. In order to account for systematic errors and an apparent small extension, we measured values for four different aperture radii $(r=0$ !" 4,0 ". 5 , 0 ! 6,0 !" 7), six different sky annuli (inner radii from $r=0$ ". 96 to 1 ." 6 , with different widths ranging from $\Delta r=0$ ". 6 to $1 . " 5$, where the latter is limited by a nearby diffraction spike). We also checked 10 slightly different aperture center positions (maximal difference $\sim 0$ ". 3). The largest systematic effect on the measured magnitude came from increasing the aperture radius from 0 ." 4 to 0 ." 6 (up to $\sim 0.3$ brighter magnitude), indicating that the source may have an

\footnotetext{
5 ISR 2016-14 Supplemental Dither Patterns for WFC3/IR, J. Anderson; http://www.stsci.edu/hst/wfc3/documents/ISRs.
}

extension of up to $r=1$ ". 2 . The effect of changing the aperture center position reached $\sim 0.1$ in magnitude. The magnitude effect of different sky annuli was much smaller ( $\sim 0.03)$. Considering these different measurements, we obtained the following median values and $1 \sigma$ uncertainties (including systematic errors): a Vega magnitude of $23.7 \pm 0.2$, and an ST magnitude of $27.1 \pm 0.2$, corresponding to a flux density of $0.40 \pm 0.06 \mu \mathrm{Jy}$.

To test the hypothesis that the source is extended, we subtracted the point-spread function (PSF) from the emission. In a drizzled image, pixels are correlated due to the drizzling process, and different PSFs are expected for individual sources due to the different sampling and signal-to-noise properties of each object (Anderson 2014). Therefore, we apply the "bundle" approach by Anderson (2014), which uses the flat-fielded FLT images derived from each of the 6 exposures. Accurate (4 times supersampled) "effective" PSFs were constructed for the FLT domain and are available at the Space Telescope Science Institute's WFC3 webpages ${ }^{6}$ (Anderson 2016). We chose 12 apparent isolated point sources as reference sources. Most of them are similarly faint as the target, but we also included a few moderately bright objects not afflicted by diffraction spikes. Using a pixel raster of $41 \times 41$, we derived bundles for the target as well as for the 12 reference sources. We created stack images of each source (i) after sky subtraction but without PSF subtraction, and (ii) after PSF and sky subtraction. Comparing these "before" and "after" images allowed us to assess the goodness of the PSF subtraction. In general, the FLT-based PSF subtraction works well. In the resulting images, residuals are visually negligible for objects as faint as the target. For brighter objects, the relative residuals (with respect to unsubtracted image) are negligible as well. We refer to the Appendix for the study of several reference sources. There are clear extended-emission residuals in the case of the target; see Figure 2, in which we also show the PSF subtraction result for a similarly faint source for comparison.

Our results led us to conclude that the NIR source at the location of RX J0806.4-4123 is definitely an extended one. The maximum extension of the target source is reached at a position angle of $\sim 20^{\circ}$ east of north. Along the line of maximum extension, the angular size of the full major axis is at least 0 ". 8. At $r=0$." 4 from the center, i.e., at a distance equal to the semimajor axis, the flux has decreased to $23 \%$ of the peak flux value (for comparison, a similar flux decrease for a reference point source, e.g., s4 in Figure 6, results in an "extension" of 0 ". 3 ). In the perpendicular direction, the full minor axis was measured to be $\approx 0$ ". 5 . If one assumes that there is indeed an additional point source, the flux of that point source represents $\sim 20 \%$ of the total F160W flux according to our PSF subtraction. In order to estimate a firm upper limit of the contribution of a possible point source to the total flux, we slightly varied the position of the subtracted point source. Using 18 positions within $r=0$ ". 15 of the maximum NIR flux, we estimated a standard deviation of $2.5 \%$ in flux. In addition, we manually increased the subtracted point-source flux from the fit value to larger ones until the residual "hole" was inconsistent with background fluctuations of the source-free region. This criterion was met when the point source constitutes $42 \%$ of the total flux. Therefore, the estimated conservative upper limit on the contribution of a point source is $50 \%$.

\footnotetext{
6 http://www.stsci.edu/hst/wfc3/documents/handbooks/currentIHB/c07_ ir07.html\#447580
} 


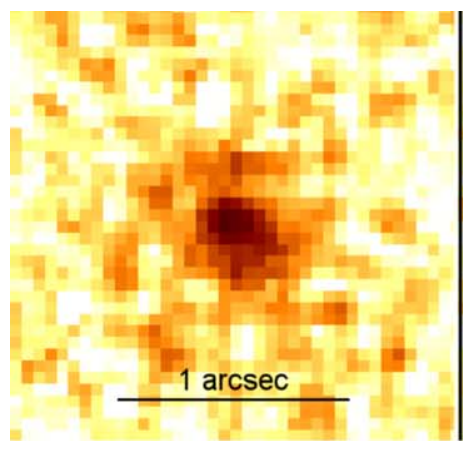

The target, $\mathrm{STmag}=27.4, \mathrm{R}_{\operatorname{maxmin}}=0.63$

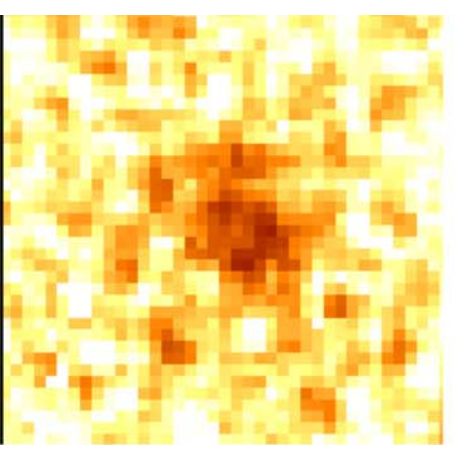

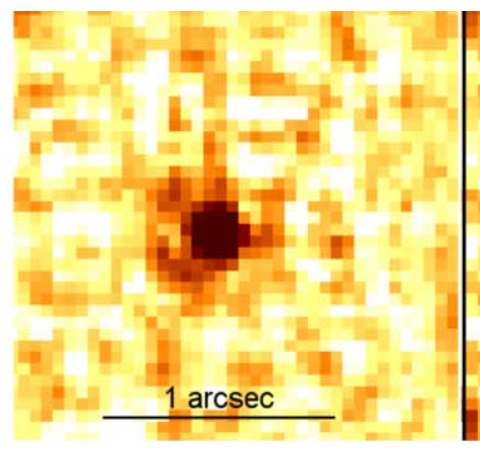

$\mathrm{s} 4, \mathrm{STmag}=27.5, \mathrm{R}_{\operatorname{maxmin}}=0.17$

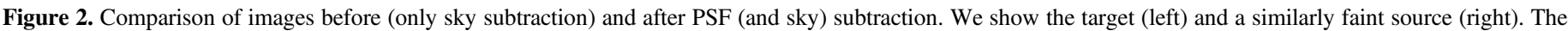

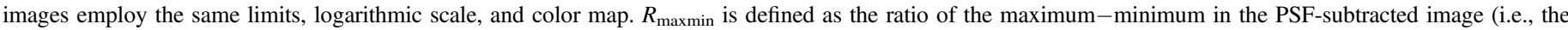

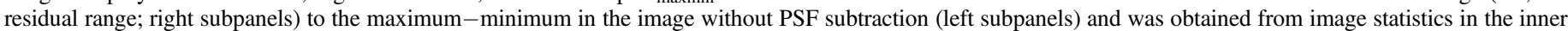

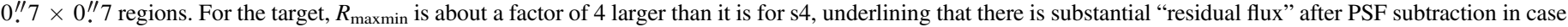
of the target. For other reference source examples in the same observation, see Figure 6.

\subsection{Gemini Observation}

RX J0806.4-4123 was observed in the $K_{s}$ band with Gemini South equipped with FLAMINGOS-2 (Eikenberry et al. 2012). The observations were carried out from 2017 January 14 to 2017 February 6 in Fast Turnaround observing mode. The program ID was GS-2016B-FT-23. We obtained 492, 12, and 226 dithered exposures with integration times of $10 \mathrm{~s}, 12 \mathrm{~s}$, and $15 \mathrm{~s}$, respectively, resulting in a total exposure time of $2.3 \mathrm{hr}$. The field of view of the Hawaii-2 array has a diameter of 6!1 and a pixel scale of 0 " 18 per pixel at the $f / 16$ telescope focal surface. The airmass of the individual exposure ranged from 1.02 to 1.32 , the average airmass was 1.08

For the data reduction, we used the $\mathrm{f} 2$ package within the Gemini IRAF Package (version 1.12) with IRAF (version 2.16). We applied the usual corrections (dark current subtraction, bad pixel mask correction, flat-fielding). After constructing sky frames with nisky, the flat-divided sky frames were subtracted from the respective flat-divided science images. Taking into account the different exposure times, we combined the data from individual nights, as well as the data from all nights. Using Gaia data release 1 (Gaia Collaboration et al. 2016a, 2016b) and the Graphical Astronomy and Image Analysis Tool (GAIA) of the JAC Starlink Project (Draper et al. 2016), we calibrated the astrometry of the reduced images to an absolute astrometric accuracy of 0 ." $13(1 \sigma)$.

We selected 16 stars (magnitudes $K_{s}=11.92-14.62$ ) from the 2MASS point-source catalog (Cutri et al. 2003) with quality flag AAA and no obvious nearby neighbors in the combined image to establish a photometry scale. We applied the daophot and photcal IRAF packages and used an aperture radius of 7 pix for the 2MASS stars (the FWHM is about 3 pix). We determined a zero-point of $25.50 \pm 0.02$. With this calibration, all but the three brightest sources (brighter than 12.35) have measured flux values within $2.2 \sigma$ of their 2 MASS magnitudes. The reason for the deviation of the brightest reference source is likely a slightly nonlinear behavior of the detector in some of the longer exposures due to imperfect screening. Based on the other 13 calibration stars, reliable $K_{s}$ magnitudes are 13.0 and fainter.

There is no significant $K_{s}$ source at the position of RX J0806.4-4123, though there may be a very faint (less than $1 \sigma$ ) flux enhancement (see Figure 3). Formally, we measured the flux in an aperture with a radius of 7 pix and obtained a magnitude $22.4 \pm 0.4(1 \sigma)$, resulting in an upper $3 \sigma$ limit of $K_{s}<21.2$ (corresponding to $<2.2 \mu \mathrm{Jy}$ ) at the position of the neutron star. Such an upper limit is consistent with $\sim 3 \sigma$ detections in the surrounding area (radius $r \sim 30^{\prime \prime}$ ), where such faint sources are found to have magnitudes of 20.8 to 21.4, depending on the varying background emission.

\section{Discussion}

The NIR source at the location of RX J0806.4-4123 is unambiguously confirmed with our HST F160W observations. The measured F160W flux, $0.40 \pm 0.06 \mu \mathrm{Jy}$, is clearly in excess of the expected neutron star flux, considering the extrapolation of its UV-optical flux (see Figure 4). In addition, the object has a size of at least 0 ."8 (Section 2) and an inhomogeneous brightness distribution, consisting of a brighter "core" and elongated "halo" emission (Figure 2). In order to estimate the likelihood of an unassociated background source, we measure a source density of 0.07 sources per square arcsec in the F160W image, which includes all sources with STmag $\leqslant 27.4$ (conservative limit since the target has STmag $=27.1 \pm 0.2$ ). The separation of the flux peak of the extended emission from the neutron star position in the 2010 HST F475W image is 0". 15. Recent Chandra observations indicate a proper motion of RX J0806.4-4123 of $\approx 20$ mas yr $^{-1}$ (B. Posselt et al. 2018, in preparation; the previous limit was $\mu<86$ mas yr$^{-1}(2 \sigma)$, Motch et al. 2009). Considering a (conservative) total position uncertainty of 0.4 (including proper motion $2 \sigma<50 \mathrm{mas} \mathrm{yr}^{-1}$, F475W position error $<0$ ". 1 ), and $N=1$ trials, we estimate a by-chance probability of $3.7 \% .^{7}$ Therefore, we regard an unassociated background source as unlikely.

Even though the by-chance probability for an unassociated background source is low, a background galaxy might explain the detected NIR emission. The optical and UV data obtained with HST (Figure 2 by Kaplan et al. 2011) do not show extended emission, and the fluxes are consistent with those measured for the other six of the Magnificent Seven, i.e., it is reasonable to assume that there is no significant flux contribution from a galaxy to the F475W and L140P flux measurements of RX J0806.4-4123. The colors of galaxies indicate that any putative galaxy counterpart must be either

\footnotetext{
Using the Chandra ACIS position from 2015 results in a larger total position uncertainty because of the larger X-ray positional error.
} 


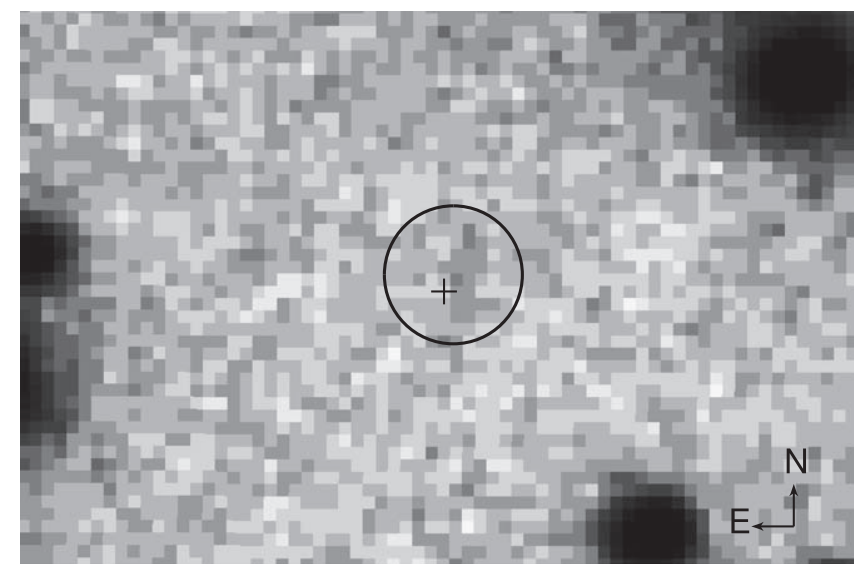

Figure 3. $2017 K_{s}$ image $\left(8^{\prime \prime} \times 12^{\prime \prime}\right)$, obtained with Gemini South equipped with FLAMINGOS-2. The circle has a radius of $1^{\prime \prime}$ and marks the F160W source at the position of RX J0806.4-4123, and the cross marks the 2002 Chandra position, as in Figure 1. For the Gemini data, the $3 \sigma$ upper limit at that position is $K_{s}<21.2$.

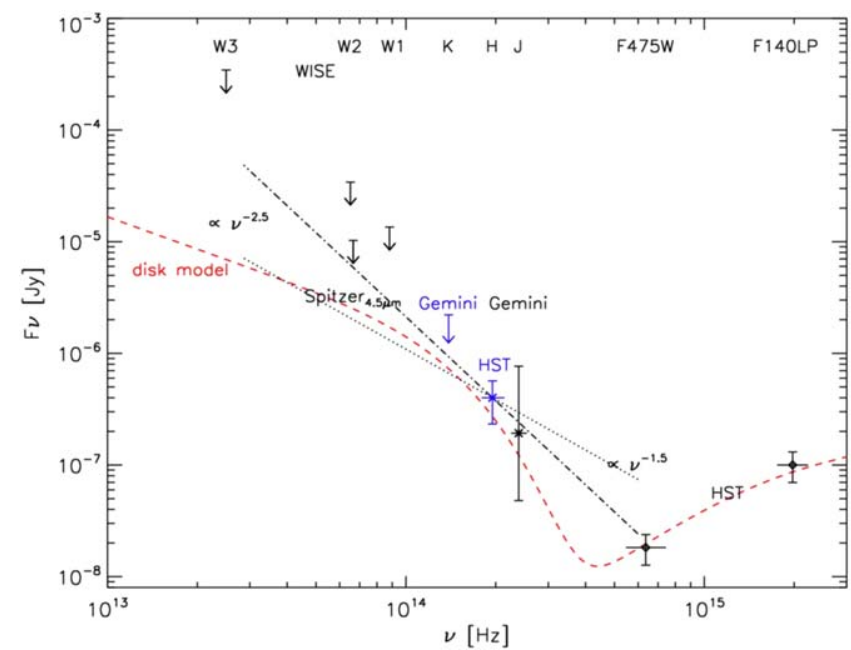

Figure 4. HST F160W flux measurement and the $3 \sigma$ upper limit in the $K_{S}$ band from the Gemini telescope are marked in blue; the other limits and measurements are from Posselt \& Luhman (2016), Posselt et al. (2014), and Kaplan et al. (2011). The shown measurement error bars indicate $3 \sigma$ uncertainties. The red dashed line shows the predicted flux of the fallback disk model discussed in Section 3.2. This is the same model that was found to fit the X-ray to optical spectrum by Ertan et al. (2017; their Figure 5). The plotted disk model was calculated for $\cos i=0.5$ where $i=60^{\circ}$ is the inclination of the observer with respect to the disk midplane normal. The NIR flux would be the same for $\cos i=0.25$ if about half of the flux comes from scattered light from the outer disk, as outlined in Section 3.2. The black dotted and dashed-dotted lines indicate different power-law spectral slopes.

very red (e.g., Daddi et al. 2000; Franx et al. 2003; van Dokkum et al. 2006; Guo et al. 2012), or, considering the lack of F475W flux, be at redshift $z \gtrsim 3.5$ (e.g., Giavalisco 2002). The same result follows from simulations using the mock galaxy catalog by Williams et al. (2018). These simulations also show that the probability of detecting galaxies with consistently red observed colors (either star-forming or nonstar-forming) in the field is low-only $\sim 0.2$ per square arcmin are expected to have F160W fluxes within the measured flux uncertainties while also being sufficiently faint in F475W. The analysis of actual, sufficiently deep observations, results in similar numbers. Analysis of the data on the Hubble Ultra Deep Field (HUDF; Rafelski et al. 2015) yield $0.39+/-0.17$ galaxies per square arcmin if red and faint sources similar to our target are considered. The uncertainty results from the large Poisson error in the very small $12.8 \mathrm{arcmin}^{2}$ field of view. ${ }^{8}$ Furthermore, we note that high-redshift galaxies are in general more compact, with smaller sizes than low-redshift galaxies (van der Wel et al. 2014; Shibuya et al. 2015). The detected $\sim 1^{\prime \prime}$ NIR extension would indicate an unusually large highredshift galaxy. In the mock galaxy simulations, for example, $<5 \%$ of galaxies with consistent observed colors are large enough to also be consistent with the detected NIR extension. Thus, we conclude that a high- $z$ galaxy is an unlikely counterpart of the extended NIR emission at the position of RX J0806.4-4123.

The detected extended emission could also be due to coincidence with emission of the interstellar medium (ISM). Scattered stellar radiation, hot dust, or line emission from the ISM gas are potential origins. We discuss these possibilities in more detail in Section 3.1.

If the NIR emission is associated with RX J0806.4-4123, then $L_{\mathrm{F} 160 \mathrm{~W}} \sim 6 \times 10^{27} \mathrm{erg} \mathrm{s}^{-1}$, assuming a distance of $250 \mathrm{pc}$. Using the X-ray flux and spin-down power of the $\mathrm{NS}$, the NIR efficiency is $\eta_{\mathrm{F} 160 \mathrm{~W}}=L_{\mathrm{F} 160 \mathrm{~W}} / \dot{E}=4 \times 10^{-3}$, and the luminosity ratio $L_{\mathrm{F} 160 \mathrm{~W}} / L_{X}=3 \times 10^{-4}$. The F160W flux value, $0.40 \pm 0.06 \mu \mathrm{Jy}$, and a size of $0 . " 5 \times 0$." 8 , translate into an average brightness temperature ${ }^{9} T_{B}=282 \mathrm{~K}$. If our source were a blackbody with this temperature, then one would expect a very high IR flux, e.g., $F_{4.5} \mu \mathrm{m}=49 \mathrm{Jy}$ which is clearly above the current flux limits by Spitzer. However, thermal emission with a temperature, $T=950 \mathrm{~K}$ and a filling factor ${ }^{10}$ of $7.3 \times 10^{-11}$ would be consistent with the F160W and $J$-band flux measurements, as well as the upper limits obtained for $K_{S}$ and $4.5 \mu \mathrm{m}$. It follows from these estimates that thermal emission is, in principle, consistent with the (N)IR measurements if the emitting area is a very small fraction of the apparent area of the detected extended source. Thermal emission could come from warm gas or X-ray irradiation and subsequent cooling of solid bodies such as dust or a substellar companion. At a distance of $250 \mathrm{pc}$, an extension of $\sim 0$ ". 8 corresponds to $3 \times 10^{15} \mathrm{~cm}$ or $200 \mathrm{au}$. This rules out a substellar companion as the sole origin of the NIR excess. However, the existence of a warm substellar companion cannot be entirely ruled out, since the conservative upper limit on the contribution of a point source to the total flux is $50 \%$. Such a flux limit translates into a substellar companion mass limit of $<10$ Jupiter masses (using a bolometric correction of 3.5 (Golimowski et al. 2004) according to the calculations presented by Burrows et al. (2001), assuming a distance of $250 \mathrm{pc}$ and an age of $10 \mathrm{Myr}$ ). Warm gas and heated dust, either in the ISM or cirumstellar matter, are discussed in Sections 3.1 and 3.2.

Alternatively, the F160W measurement can also be explained by non-thermal emission. Assuming a power-law $F_{\nu} \propto \nu^{\alpha}$ for non-thermal emission, the current spectral constraints imply either a rather steep slope $\alpha<-2.5$, or an exponential cutoff between the F160W and F475W bands. Charged particles producing curvature and/or synchrotron

\footnotetext{
8 The GOODS (e.g., Skelton et al. 2014) fields are larger, but the respective F475W observations are shallower than those of RX J0806.4-4123.

9 The brightness temperature $T_{B}$ is defined from the equation $F_{\nu} \Omega^{-1}=$ $B_{\nu}\left(T_{B}\right)$, where $F_{\nu}$ is the flux density measured in the solid angle $\Omega$ subtended by the source, and $B_{\nu}\left(T_{B}\right)$ is the Planck function.

${ }^{10}$ The filling factor is the ratio of projected emitting area and projected source area.
} 
emission would be the likely source. With respect to an association to a neutron star, the most obvious origins are magnetospheric emission (for the possible flux contribution from a point source) or a pulsar wind and the shocks it produces. We discuss such a PWN in Section 3.3.

\subsection{Emission from the ISM?}

In principle, an ISM density enhancement along the line of sight could scatter NIR emission from ambient light and produce the observed extended NIR emission. The so-called cloudshine is a large-scale (typically, several arcminutes) phenomenon that is caused by ambient star light around large and dense molecular clouds (Lehtinen \& Mattila 1996; Foster $\&$ Goodman 2006). There are no other extended NIR features and no indication of a molecular cloud (e.g., Dame et al. 2001; Dobashi et al. 2005) in the field of RX J0806.4-4123, which one would expect if the NIR emission were due to cloudshine.

Instead of the interstellar radiation field and a large, dense molecular cloud, one could postulate that the NIR emission could originate from local point-source emission that is scattered by a small (i.e., unaccounted for) ISM enhancement at a slightly larger distance than the one of RX J0806.4-4123. ${ }^{11}$ However, in this case the expected thermal NIR flux from the XTINS surface alone is too low to produce the large observed NIR excess by scattering. An additional NIR emitter would be required, as well as an unlikely "wall" in the ISM.

If the NIR excess flux is due to genuine emission from the ISM, it could originate either from ISM gas or dust in the diffuse ISM or in an ISM density enhancement. There are a few emission lines of ISM gas in the $H$ and the other NIR-bands. Strongly forbidden rotational-vibrational emission lines of $\mathrm{H}_{2}$ are observed, for example, in reflection nebulae or shocked regions in star-forming regions and around AGB stars (e.g., Shull \& Beckwith 1982). These $\mathrm{H}_{2}$ emission lines are due to collisional excitation or UV fluorescence, and in accordance with their detection in dense environments they require high densities and significant molecular fractions (Black \& van Dishoeck 1987; Hollenbach \& McKee 1989). The low $N_{\mathrm{H}}$ value of RX J0806.4-4123 implies not only a low density, but also a very low molecular fraction, $2 N_{\mathrm{H}_{2}}\left(2 N_{\mathrm{H}_{2}}+N_{\mathrm{H}}\right)^{-1}<10^{-4}$, because inefficient self-shielding from cosmic rays leads to dissociation of $\mathrm{H}_{2}$ (Savage et al. 1977; Burgh et al. 2007). Therefore, the NIR excess flux around the neutron star is unlikely to be produced by $\mathrm{H}_{2}$ emission lines.

Interstellar dust emission peaks in the far-infrared. It can also emit NIR photons if it is hot enough. The required high temperatures (1000-2000 K) in thermal equilibrium could only be reached very close to the XTINS (unresolved by HST) by typical (size $\sim 0.1 \mu \mathrm{m}$ ) dust grains. A non-equilibrium process, the so-called stochastic heating of very small grains $\lesssim 10 \AA$ or large polycyclic aromatic hydrogen $(\mathrm{PAH})$ molecules by UV photons, was found to produce the large-scale NIR emission of reflection nebulae (e.g., Sellgren 1984; Draine \& Li 2001). There are two reasons why very small grains/PAH molecules

\footnotetext{
11 The absorbing hydrogen column density inferred from the X-ray spectrum of RX J0806.4-4123 is between $N_{\mathrm{H}}=(0.4 \pm 0.1) \times 10^{20} \mathrm{~cm}^{-2}$ (Haberl et al 2004) and $N_{\mathrm{H}}=(1.7 \pm 0.2) \times 10^{20} \mathrm{~cm}^{-2}$ (Kaplan \& van Kerkwijk 2009), depending on the spectral model. Thus, using the relation between $N_{\mathrm{H}}$ and $A_{\mathrm{V}}$ by Foight et al. (2016), the extinction of the neutron star, $A_{\mathrm{V}}<0.1$, rules out a residence in dense ISM. The color excess along the line of sight toward the neutron star is only $E(B-V)=0.14 \pm 0.08(1 \sigma)$ even at a distance of $1.5 \mathrm{kpc}$ (well beyond the likely distance of the XTINS) according to a recent 3D map of the ISM by Capitanio et al. (2017) (their spatial resolution along the line of sight is $\sim 200 \mathrm{pc}$ at $1-1.5 \mathrm{kpc}$ ).
}

of the ISM cannot be responsible for the extended emission around RX J0806.4-4123. First, there is not a sufficient amount of very small grains in the ISM surrounding the XTINS to produce the observed NIR flux. Using the constraints on the XTINS spectrum in UV (Kaplan et al. 2011), and our flux measurement at $1.6 \mu \mathrm{m}$, one can estimate the required number density, $n_{\mathrm{vsg}}$, of very small grains $(\sim 10 \AA)$, which are stochastically heated by $\sim 10 \mathrm{eV}$ UV photons (Sellgren 1984). The value, $n_{\mathrm{vsg}} \sim 10^{-3} \mathrm{~cm}^{-3}$, is at least four orders of magnitude larger than what one can expect for the typical ISM with a hydrogen number density $n_{\mathrm{H}} \sim 1 \mathrm{~cm}^{-3}$, dust-togas ratio of $6 \%$, and typical grain size and composition distribution (all from, e.g., Zubko et al. 2004; we used their BARE-GR-S dust model, which matches observations of the ISM in the solar neighborhood). The lack of known "NIR halos" around other strong UV sources such as white dwarfs supports our estimate that the diffuse ISM does not provide enough small grains for substantially enlarged NIR flux close to these objects. The second reason why very small ISM grains are an unlikely origin for the observed NIR emission is the strong X-ray emission of the XTINS. Voit (1991) estimated that X-ray photons efficiently evaporate grains of sizes $\lesssim 10 \AA$. Overall, we conclude that the observed NIR emission around RX J0806.4-4123 is unlikely to come from either the gas or dust of the photon-heated ISM. We discuss the interaction of potential pulsar wind particles with the ISM in Section 3.3.

\subsection{Emission from Circumstellar Material?}

If the NIR emission comes from the immediate vicinity of RX J0806.4-4123, it could be due to a surrounding disk or torus structure, similar to what has been inferred for the magnetar $4 \mathrm{U} 0142+61$ from the Spitzer detection by Wang et al. (2006) or for white dwarfs from NIR detections (e.g., Melis et al. 2010). The stochastic heating of very small grains, which was discussed in the previous section for the ISM, is a possibility for circumstellar matter, too. However, close to a neutron star, one expects quick destruction (evaporation by X-rays) and removal (by Poynting-Robertson drag; e.g., Figure 10 by Posselt et al. 2014) of such small grains. Hence, regular replenishment, e.g., by collisions of asteroids or large grains, would be required to keep such a "debris disk" bright in the NIR.

Considering an active fallback disk model, Ertan et al. (2017, 2014) showed that such a disk is able to produce the X-ray properties and the optical excess of the XTINSs. Here, we use their model to check whether such a fallback disk can reproduce the measured NIR excess. The model employs dissipative heating, which is most relevant in the active inner part of the disk, for RX J0806.4-4123, from the inner disk radius, $r_{\text {in }} \sim 10^{9} \mathrm{~cm}$ to $\lesssim 5 \times 10^{11} \mathrm{~cm}$. The model also considers X-ray irradiation, which is most relevant for the passive outer part of the disk. For details on the model and the model parameters for RX J0806.4-4123, we refer to Ertan et al. $(2017,2014)$. The outer radius of the passive disk is not well known and depends on the initial disk conditions and the X-ray luminosity in the early phase of evolution. However, the local irradiation temperature is very small for large radii-for RX J0806.4-4123, it decreases to $<30 \mathrm{~K}$ for $r>10^{13} \mathrm{~cm}$. For studies of the flux at infrared and shorter wavelengths, it is sufficient to use an outer radius $r_{\text {out }} \sim 10^{13} \mathrm{~cm}$.

The extension of the NIR source at the distance of RX J0806.4-4123 is $r \sim 10^{15} \mathrm{~cm}$ (or $\sim 100 \mathrm{au}$ ). This raises 
the question of whether there is any possibility of the disk model explaining not only the observed NIR flux but also the observed source extension. Drawing on the findings in protoplanetary disk studies, favorable properties of the disk and viewing geometry can perhaps provide such an explanation. For protoplanetary disks, it was shown that in the case of a flared disk NIR emission can be scattered and produce extended emission (e.g., Mulders et al. 2013; Anthonioz et al. 2015). First, we check whether the disk model can reproduce the observed spectral energy distribution. Then we outline a potential explanation for the observed source extension.

Using $r_{\text {in }}=10^{9} \mathrm{~cm}$ and $r_{\text {out }}=10^{13} \mathrm{~cm}$, we obtain the model spectrum given in Figure 4. The disk model curve in Figure 4 is within the error range of the data, illustrating that the fallback disk model can reproduce the observed NIR flux. Within this disk model, the work done by the magnetic torque heats the inner rim of the disk, which then produces the optical and UV fluxes. In contrast, the NIR emission of the model spectrum is produced from the surface of the irradiated inner disk within a few times $10^{9} \mathrm{~cm}$ of the XTINS. One implication is that a completely passive disk could also produce the NIR emission, but not the optical-UV emission.

One possible way to explain the observed source extension is based on the assumption of disk flaring and scattering off the outer passive disk surface. The thickness of a disk can be described with the pressure scale height, $h \simeq c_{\mathrm{s}} / \Omega_{\mathrm{K}}$, where $c_{\mathrm{s}}$ is the sound speed and $\Omega_{\mathrm{K}}$ is the local Keplerian angular velocity of the disk. In flared disks the flaring is described by the aspect ratio, $h / r$. The outer radii of protoplanetary disks around T Tauri stars reach several hundred au and the $h / r$ values can approach unity (see, e.g., Chiang \& Goldreich 1997; Armitage 2010; Dullemond \& Monnier 2010 for reviews). Chiang \& Goldreich (1997) (CG97) calculated self-consistently the aspect ratio and the temperature profiles of these disks. We follow the result of CG97 for our estimates, but adapt the model parameters (luminosity, mass) to the properties of RX J0806.4-4123. CG97 found $h / r \propto r^{2 / 7}$ for an optically thick inner disk region. Outside this region there is a radially isothermal optically thin region along which the aspect ratio increases even faster with increasing $r$ as $h / r \propto r^{1 / 2}$. The disk photosphere, defined as the disk surface layer corresponding to the optical depth of unity to the irradiating flux, is estimated by CG97 to be $H \simeq 4 h$. In the luminosity regime of RX J0806.4-4123, $L_{*} \sim L_{X}=2 \times 10^{31} \mathrm{erg} \mathrm{s}^{-1}$; using $h / r \propto L_{*}^{1 / 7} M^{-4 / 7}$ (CG97) with $M=1.4, M_{\odot}$, we estimate the aspect ratio of the disk for RX J0806.4-4123 as $H / r \simeq 2.7 \times 10^{-2} r_{\mathrm{au}}^{2 / 7}$, where $r_{\mathrm{au}}$ is the radial distance in au. For $r_{\mathrm{au}}=100$, we find $H / r \simeq 0.1$. This could be taken as a lower limit, since the outermost part of the passive disk could be optically thin to its own emission, along which $H$ increases faster with increasing $r$, as $r^{1 / 2}$. In CG97, the radially isothermal disk region has a temperature $\sim 20 \mathrm{~K}$. For RXJ0806.4-4123, such a temperature is reached at $r \sim 8 \times 10^{12} \mathrm{~cm}$. Thus, a large fraction of the outer passive disk of RXJ0806.4-4123 could be radially isothermal with $H / r \propto r^{1 / 2}$. In this case, the result is $H / r \approx 0.3$ at $100 \mathrm{au}$. Hence, depending on the extension of the radially isothermal region, $H / r$ could be between 0.1 and 0.3 at the outermost disk. For our estimates below we take $H / r=0.2$ at $r_{\text {out }} \simeq 100 \mathrm{au}$.

The NIR luminosity illuminating and being scattered off the outer disk surface, $L_{\text {scat }}$, is a small fraction of the total NIR luminosity emitted from the inner disk, $L_{\text {in }}$. As seen from the inner disk, the outer passive disk subtends a solid angle $\Omega_{\mathrm{d}}$ from the midplane of the disk $\left(\theta=90^{\circ}\right)$ to the surface of the outer disk $\left(\theta=\theta_{\mathrm{d}}\right)$. For $H / r \simeq 0.2$ at $r \simeq 100 \mathrm{au}$, this angle is $\theta_{\mathrm{d}} \simeq 78^{\circ}$. Assuming an albedo of $\beta \simeq 0.9$, we estimate that up to $4 \%$ of the total NIR emission from the inner disk could illuminate and be scattered by the outer disk surface, $L_{\text {scat }} / L_{\text {in }} \lesssim 0.04$.

The observed ratio of the NIR flux scattered off the outer disk to the NIR flux from the inner disk $\left(r \sim 10^{9} \mathrm{~cm}\right)$ depends on the details of the viewing and the scattering geometry, the actual $h / r$ profile, temperature, and the extension of the currently passive disk. For instance, the scattering geometry matters because the scattered emission is unlikely to be purely isotropic. The angular distribution of photons scattered by a dust grain depends on the grain composition and grain size (Draine 2003; Mulders et al. 2013). Large grains in particular show very anisotropic scattering, where one scattering produces a conical beam with a small solid angle $\Omega_{s c}$. The NIR light emitted from the inner disk is estimated to be scattered from a thin layer of the disk surface. In order to estimate the contribution of the scattered component to the observed NIR flux, we use a simplified picture and represent the total scattered emission by two narrow fan beams opening from the two surfaces of the disk. In the case of single scatterings, the solid angle of one fan beam can be estimated by integration over the azimuthal angle as $\Omega_{\text {beam }} \approx 2 \pi^{1 / 2} \Omega_{s c}^{1 / 2}$. Inside this solid angle, the ratio of the observed scattered flux to the observed inner disk flux, $F_{\text {scat }} / F_{\text {in }}$, is related to the ratio of the luminosities as

$$
\frac{F_{\text {scat }}}{F_{\text {in }}} \simeq \frac{\left(2 \pi / \Omega_{\text {beam }}\right)}{\cos i} \frac{L_{\text {scat }}}{L_{\text {in }}},
$$

where $i$ is the inclination angle between the line of sight and the normal to the midplane of the disk. If we assume, for example, $\Omega_{\text {beam }} \sim 1 \mathrm{sr}$, and $\cos i=0.25$ (i.e., $i \simeq 75^{\circ}$ ), we obtain $F_{\text {scat }} / F_{\text {in }} \approx 1$ and $F_{\text {in }} / F_{\text {tot }} \approx 0.5$, which are consistent with the observation. For this scenario, half of the $H$-band emission is produced at the (unresolved) inner disk, while the remaining half is the contribution from the scattered light. In this example, the inclination of the observer and the inclination of the scattering disk surface are very similar, and the observer remains inside the beaming solid angle of the near-side disk surface. Instead of the simple fan-beam geometry for the scattered NIR radiation, the NIR light could actually be scattered in all directions. Our explanation remains valid if the scattered flux density is still concentrated near the disk surface. If the disk is observed at small inclination angles (close to face-on view), the contribution of the scattered light to the observed flux decreases depending on the efficiency of the "beaming," while the flux from the inner disk increases. In other words, the observed manifestations of any large disks around XTINSs could vary significantly, depending on the optical properties of the disks, the inclination $\cos i$, and the observed wavelength.

Equation (1) shows that for an observer inside the solid angle of the beamed scattering, $\Omega_{\text {beam }}$, the observed $F_{\text {scat }} / F_{\text {in }}$ could be significantly greater than $L_{\text {scat }} / L_{\text {in }}$. In our simplistic model of an assumed scattering anisotropy, the observer sees not the entire disk but only a fraction of the disk surface close to the line of sight, which, at a given outer radius, reduces the size of the observed extended emission in comparison to the actual size of the disk. However, the observed size increases with 
increasing contribution from multiple scatterings. A given light ray can go through multiple scatterings, both local and at different radii, which can significantly change its azimuthal direction. The details of the extended emission depend on the actual geometry and the scattering properties of the disk and require detailed modeling to quantify the "beaming" of the NIR emission and its observable azimuthal distribution. We also note that we extrapolated the results from protoplanetary disks to our case of a potential neutron star disk. The composition of such a disk is very likely quite different from that of a protoplanetary disk. For example, one could expect a higher metallicity and possibly a higher dust-to-gas ratio in supernova fallback material.

In addition to thermal continuum, such a disk could also show line emission. Modeling of different disk geometries, densities, and compositions is beyond the scope of this paper, in particular because we currently have a good measurement in only one photometric band of the (N)IR.

As an alternative to a disk, scattering of the emission from the inner disk by the dust grains in an additional outer cold dusty belt or torus structure around RX J0806.4-4123 could also explain the extended NIR emission. Our previous Herschel $160 \mu \mathrm{m}$ detection for the region of RXJ0806.4-4123 resulted in an estimate of dust grain locations at $r_{d}=2.3 \times 10^{16} a_{\mu \mathrm{m}}^{-1 / 2} \mathrm{~cm}$ to $2.2 \times 10^{15} a_{\mu \mathrm{m}}^{-1 / 2} \mathrm{~cm}$ for a reasonable dust temperature range of $10-22 \mathrm{~K}$ and in dependence of the grain size, $a_{\mu \mathrm{m}}$, in $\mu \mathrm{m}$ (Posselt et al. 2014). We had speculated about the existence of a belt structure to explain the large ratio of the luminosity at $160 \mu \mathrm{m}$ to the XTINS' X-ray luminosity (for details, see Posselt et al. 2014). The association of the Herschel $160 \mu \mathrm{m}$ detection with RX J0806.4-4123 is not as firm as the Hubble NIR detection because of the worse spatial resolution of Herschel. Nevertheless, it is intriguing that the spatial dimensions of the potential torus are of about the same order as the NIR extension. A dusty torus alone cannot explain the NIR emission because the scattered NIR emission from RX J0806.4-4123 would be expected to be much fainter. The implied two-component structure is not uncommon for debris disks around main sequence stars (e.g., Su et al. 2013) and may also be a possibility around a neutron star.

Overall, a disk can be the source of the detected NIR flux, while the observed source extension can be due to scattering by cold dust grains located at larger separations from the neutron star, either in a disk with favorable properties or in a surrounding dusty torus.

\subsection{A Pulsar Wind Nebula?}

Extended emission around a pulsar can also be a PWN. Most PWNe are known from high-energy and radio observations (e.g., Reynolds et al. 2017). About a dozen have been detected in $\mathrm{H}_{\alpha}$ (Brownsberger \& Romani 2014), and two have recently been discovered in the ultraviolet (e.g., Rangelov et al. 2017). A few high-energy PWNe were also detected in the (near-) infrared, e.g., the PWNe of Vela (Shibanov et al. 2003) and PSR B0540-69 (Mignani et al. 2012), but there is only one serendipitous discovery of an infrared-only PWN with Spitzer at wavelengths longer than $8 \mu \mathrm{m}$ (Wang et al. 2013). The NIR emission around RX J0806.4-4123 could come either from line emission in the shocked ambient medium or from synchrotron emission of the shocked pulsar wind. In the case of a shocked ambient medium, possible origins are emission lines of $\mathrm{H}_{2}$ and [Fe II]. These shock-excited lines are frequently observed in the dense environments of star-forming regions (e.g., Herbig-Haro objects), evolved stars with eruptive mass loss, and supernova remnants (e.g., Reipurth \& Bally 2001; Lee et al. 2014). Such a dense environment, however, is unlikely for RX J0806.4-4123 based on the X-ray absorption in the XTINS spectrum and 3D models of the ISM (Section 3.1). Hence, in the case of the PWN scenario, the emission is probably due to synchrotron emission from the shocked pulsar wind.

At low $\dot{E}$, particles cannot be accelerated to sufficiently high energies to produce X-ray emission, but emission at lower energies could be possible. According to Reynolds et al. (2017), the maximum synchrotron photon energy $E_{\max }$ of the accelerated electrons in the shocked pulsar wind can be estimated as

$$
E_{\max } \lesssim 0.13 \zeta \dot{E}_{30} B_{-6} \frac{\sigma}{\sigma+1} \mathrm{eV},
$$

where $\zeta \sim 1$ is a numerical factor, $\dot{E}_{30}$ is the spin-down power in units of $10^{30} \mathrm{erg} \mathrm{s}^{-1}, B_{-6}$ is the magnetic field at the location of the shocked pulsar wind in units of $\mu \mathrm{G}$, and $\sigma$ is the magnetization, which is defined as the ratio of the Poynting flux to the particle enthalpy flux. Expected PWN magnetic field values are of the order of $10-100 \mu \mathrm{G}$ depending on distance, while $\sigma$ is unknown but usually assumed to be $\lesssim 1$ or even $\sigma \ll 1$ (Kennel \& Coroniti 1984; Reynolds et al. 2017). The spin-down power of RX J0806.4-4123 is $\dot{E}_{30}=1.6$ (Kaplan \& van Kerkwijk 2009). Thus, $E_{\max } \sim 1 \mathrm{eV}$ (corresponding to $\lambda \approx 1.2 \mu \mathrm{m}$ ) is possible for $\sigma \lesssim 1$.

The X-ray efficiencies, $\eta_{X}=L_{X} / \dot{E}$, of observed PWNe vary greatly, reaching values between $\lesssim 10^{-6}$ and $10^{-2}$ (Reynolds et al. 2017). The similarly defined NIR efficiency of RX J0806.4-4123, $\eta_{\mathrm{F} 160 \mathrm{~W}}=4 \times 10^{-3}$, is within the PWN efficiency range seen at other wavelengths. For a pulsar moving supersonically with a total velocity $v_{\mathrm{PSR}}$ through the ambient medium with density $\rho_{\mathrm{a}}$, the stand-off radius $R_{s}$ of the bow shock apex can be described by $R_{s}^{2}=\dot{E}_{\mathrm{PW}} f\left(4 \pi c \rho_{\mathrm{a}} v_{\mathrm{PSR}}^{2}\right)^{-1}$, where $\dot{E}_{\mathrm{PW}}=\xi_{w} \dot{E} \lesssim \dot{E}$ is the fraction of spin-down power carried away by the wind, and the factor $f$ takes into account a possible anisotropy of the pulsar wind ( $f=1$ for an isotropic wind). Using an observed source extension radius $r \sim 0$ ". 4, which corresponds to $R_{s} \sim 1.5 \times 10^{15} \mathrm{~cm}$ (Section 2), $\xi_{w}$ $f \lesssim 1$, and $\rho_{\mathrm{a}} \approx n_{\mathrm{a}} m_{\mathrm{p}}$ ( $m_{\mathrm{p}}$ is the mass of a proton), we obtain

$$
n_{\mathrm{a}}^{1 / 2} v_{\perp} \lesssim 12\left(R_{S} / 10^{15} \mathrm{~cm}\right)^{-1} \mathrm{~km} \mathrm{~s}^{-1} \mathrm{~cm}^{-3 / 2},
$$

where $v_{\perp}$ is the transverse velocity component. The proper motion of RX J0806.4-4123 is unusually low, $\mu<86$ mas yr $^{-1}$ $(2 \sigma)$, with recent Chandra observations indicating $\approx 20{\text { mas } \mathrm{yr}^{-1}}^{-1}$ (B. Posselt et al. 2018, in preparation). At a distance of $250 \mathrm{pc}$, these values correspond to $v_{\perp}<100 \mathrm{~km} \mathrm{~s}^{-1}(2 \sigma)$ and $24 \mathrm{~km} \mathrm{~s}^{-1}$, respectively. This does not significantly exceed the speed of sound in the typical diffuse ISM $\left(c_{s} \sim 10 \mathrm{~km} \mathrm{~s}^{-1}\right.$ for $T \sim$ $8000 \mathrm{~K})$. The constraint on the velocity and number density in Equation (3) depends on the actual $R_{s}$, but it is uncomfortably low, particularly considering that an anisotropic wind and a typically assumed fraction of the spin-down power emitted with the wind $\left(\xi_{w} \sim 0.5\right)$ will lower the value further. $R_{s}$ could, however, be smaller, since it is difficult to establish the exact 
location of the pulsar in the extended NIR emission. Hence, a PWN can currently not be ruled out as a possible origin of this emission.

If RX J0806.4-4123 were a usual rotation-powered pulsar, the PWN would be an obvious explanation. Such an explanation is, however, somewhat surprising for a member of the Magnificent Seven, which show only thermal X-ray emission and no radio or $\gamma$-ray emission. A part of the detected NIR emission (the point-source contribution that can be at maximum $50 \%$ ) could in principle come from the magnetosphere of RX J0806.4-4123. However, the optical-UV spectrum of the neutron star, $F_{\nu} \propto \nu^{\alpha}$, with $\alpha=1.62 \pm 0.20(1 \sigma)$ (Kaplan et al. 2011), is consistent with, and thus likely dominated by, a Rayleigh-Jeans tail of thermal emission (for which $\alpha=2$ ). If there is a PWN, then a lack of detectable nonthermal emission from the neutron star at higher energies than NIR is unprecedented. It is noteworthy that the XTINS spectra of other members of the Magnificent Seven, in particular RBS $1774,{ }^{12}$ show much stronger deviation from a RayleighJeans tail, $\alpha=0.53 \pm 0.08(1 \sigma)$ (Kaplan et al. 2011). However, as discussed by Kaplan et al. (2011), a magnetospheric origin of the deviations from Rayleigh-Jeans is not the only possible explanation, in particular considering the high optical luminosities of these XTINSs. The current uncertainties in the models of strongly magnetized neutron star atmospheres at long wavelengths could be, for example, another explanation for the unusual spectra of the Magnificent Seven. If the extended NIR emission around RX J0806.4-4123 is indeed a PWN, it could indicate an interesting new avenue to study the properties of pulsar winds without very energetic particles originating from the (inner) magnetosphere. It is interesting that in comparison to typical spectral slopes of X-ray-detected PWNe, $\alpha_{\mathrm{X}} \approx-0.5$ (Kargaltsev \& Pavlov 2008), the slope of the NIR PWN, $\alpha_{\mathrm{PWN}} \lesssim-2.5$, is rather steep according to Figure 4. Such a steep slope could be explained if the NIR photons correspond to the highest electron energies in the PWN.

\subsection{Is $R X$ J0806.4-4123 Special?}

RX J0806.4-4123 has a very low transverse velocity. Perhaps it also has a viewing geometry favorable for the detection of the fallback disk. Could these properties be the reason why we have seen extended NIR emission only for this neutron star so far? Looking at the Magnificent Seven members with comparably deep NIR limits, we compare the two brightest and best studied members with RX J0806.4-4123. RX J1856.6-3754, at a parallactic distance of $123_{-15}^{+11} \mathrm{pc}$, has a transverse velocity of $192_{-28}^{+17} \mathrm{~km} \mathrm{~s}^{-1}$ and $\dot{E} \sim 3 \times 10^{30} \mathrm{erg} \mathrm{s}^{-1}$, and its $H$-band limit is 21.5 mag (van Kerkwijk \& Kaplan 2008; Posselt et al. 2009; Walter et al. 2010; Tetzlaff et al. 2011). It also has a rather puzzling $\mathrm{H}_{\alpha}$ nebula (van Kerkwijk \& Kulkarni 2001; van Kerkwijk \& Kaplan 2008; Brownsberger \& Romani 2014). RX J0720.4-3125, at a parallactic distance of $280_{-85}^{+210} \mathrm{pc}$, has a transverse velocity of $143_{-44}^{+108} \mathrm{~km} \mathrm{~s}^{-1}$ and $\dot{E}=4.7 \times 10^{30} \mathrm{erg} \mathrm{s}^{-1}$, and its $H$-band limit is 23.1 mag (Kaplan \& van Kerkwijk 2005; Posselt et al. 2009; Tetzlaff et al. 2011). In comparison to the Vega magnitude of $23.7 \pm 0.2$ measured for RX J0806.4-4123 with WFC3/F160W, the $H$-band flux limits are shallower for RX J0720.4-3125 (factor 2) and RXJ1856.6-3754 (factor 8). The smaller distance of

\footnotetext{
12 Coincidentally, RBS 1774 is the other XTINS where there is a potential Herschel detection (Posselt et al. 2014).
}

RX J1856.6-3754 is not small enough to counterbalance the effect of its shallower observation, while for RX J0720.4-3125 the large distance uncertainty makes any NIR luminosity limit highly uncertain. Thus, there is currently no indication that the NIR emission around RXJ0806.4-4123 is exceptional among the Magnificent Seven.

Reports of NIR-detected PWNe (or disks) are rare for other isolated neutron stars. Rotation-powered pulsars and magnetars are detected in the NIR, but there is neither such a steep slope between the optical and the NIR fluxes, nor are the PWNe only detected at these wavelengths (e.g., Durant et al. 2004; Durant \& van Kerkwijk 2005; Danilenko et al. 2011). Magnetars are on average at several kiloparsecs, hence any small-sized extended emission would be difficult to establish. An example of a nearby rotation-powered pulsar is Geminga, which is similarly distant $\left(d=250_{-80}^{+230} \mathrm{pc}\right)$ as RX J0806.4-4123, but has a transverse velocity of $\approx 211 \mathrm{~km} \mathrm{~s}^{-1}$ and a much higher spin-down power $\dot{E}=3.3 \times 10^{34} \mathrm{erg} \mathrm{s}^{-1}$ (Bertsch et al. 1992; Faherty et al. 2007; Verbiest et al. 2012). As Figure 6 by Danilenko et al. (2011) shows, Geminga's optical-IR spectrum is relatively flat in $F_{\nu}$, though interestingly there may be some indication of a rise toward the mid-infrared. Geminga has a prominent X-ray PWN, and Shibanov et al. (2006) reported a detection of the PWN bow shock in the $I$ band, but not in the $H S T$ NICMOS F160W image. From the higher $\dot{E}$ of Geminga, one would expect PWN emission at shorter wavelengths (Equation (2)). The situation could be different for rotationpowered pulsars with $\dot{E}$ as low as those of the Magnificent Seven. The old PSR J0108-1431 has $\dot{E}=5.8 \times 10^{30} \mathrm{erg} \mathrm{s}^{-1}$, which is only slightly larger than the value of RX J0806.4-4123. At a parallactic distance of $240_{-61}^{+124} \mathrm{pc}$, its transverse velocity is $194_{-51}^{+104} \mathrm{~km} \mathrm{~s}^{-1}$ and its $H$-band limit is 21.4 (Deller et al. 2009; Posselt et al. 2009). Again, the relatively shallow observations do not allow a meaningful comparison with RX J0806.4-4123.

\section{Conclusions}

The extended NIR emission around the isolated neutron star RX J0806.4-4123 is unlikely to come from an unrelated field object. It can be interpreted as coming either from a disk with a favorable viewing geometry or a PWN created by shocked pulsar wind particles of relatively low energy. A flux contribution of up to $50 \%$ could come from a point-like source, which could represent the unresolved inner disk or the pulsar magnetosphere in the respective interpretations. The spectra and the details of the spatial shape are expected to be quite different for the two explanations and can be probed with future high-resolution observations with the James Webb Space Telescope.

Most pulsars do not have deep NIR observations, mostly because the emission of the neutron stars at these wavelengths was expected to be just an unexciting extension of the opticalUV spectral slope. RX J0806.4-4123 is a good example that neutron stars keep surprising us. Whether the detected extended emission around RX J0806.4-4123 is a lucky coincidence of low pulsar speed and favorable viewing geometry or this is a common property among (some types of) pulsars needs to be checked with systematic deep NIR surveys.

We thank the referee, Marten van Kerkwijk, for his comments and suggestions, which helped to improve the quality of this manuscript. We are very thankful to Jay 


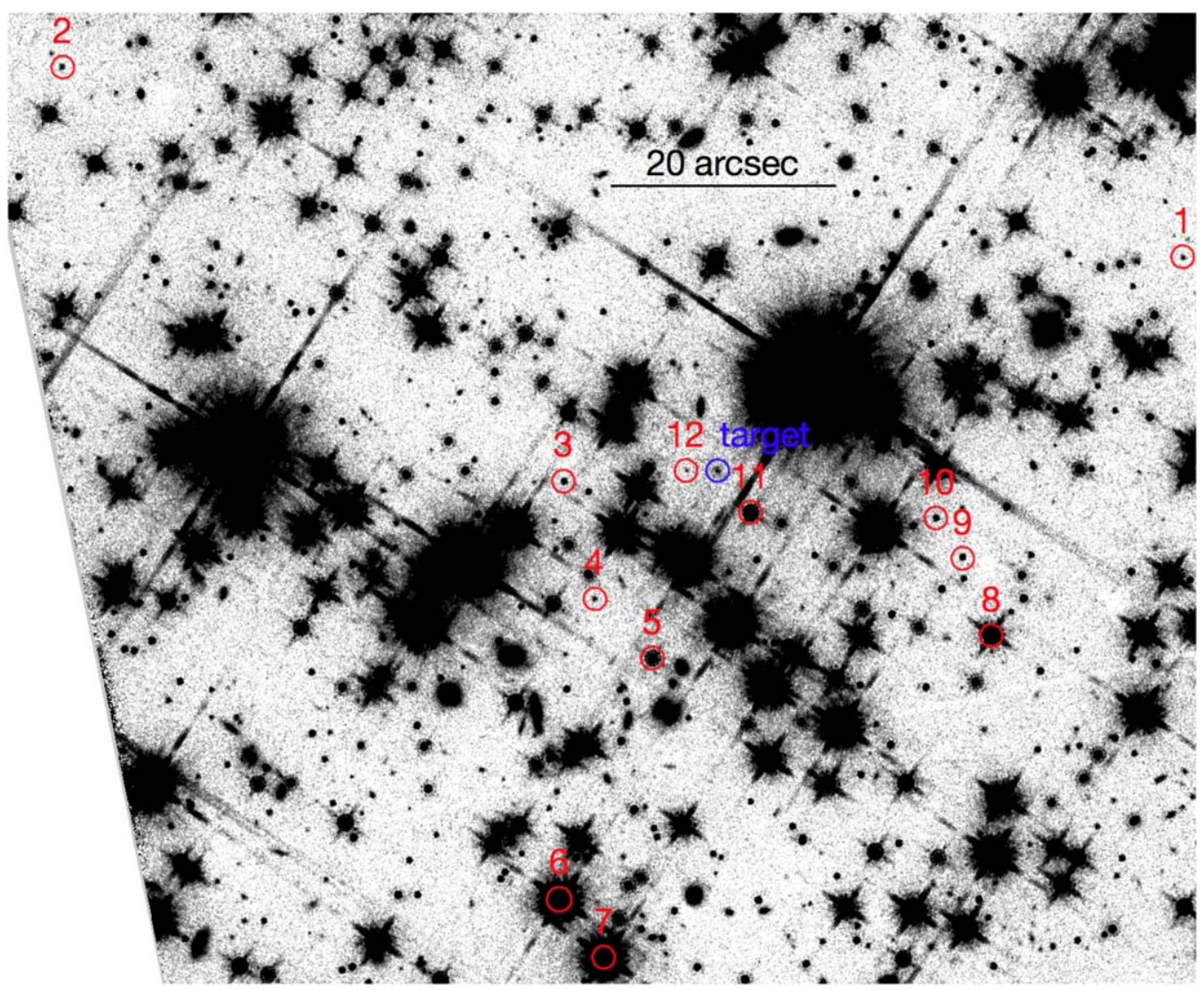

Figure 5. Location of the reference sources in the WFC3/F160W observation. North is up and east is to the left.

Anderson for his helpful support in the WFC3/F160W data reduction, in particular his crucial help with respect to an optimal PSF subtraction. We also thank Mario Gennaro and Howard Bond for helping us to optimize our observation setup.

B.P. acknowledges support for this work (program 14745) by NASA through a grant from the Space Telescope Science Institute, which is operated by the Association of Universities for Research in Astronomy, Inc., under NASA contract NAS 5-26555.

Ü.E. acknowledges research support from TÜBİTAK (The Scientific and Technological Research Council of Turkey) through grants 117F144 and and from Sabanc1 University.

C.C.W acknowledges support from the National Science Foundation Astronomy and Astrophysics Fellowship grant AST-1701546.

The Center for Exoplanets and Habitable Worlds is supported by the Pennsylvania State University, the Eberly College of Science, and the Pennsylvania Space Grant Consortium.

This work has made use of data from the European Space Agency (ESA) mission Gaia (https://www.cosmos.esa.int/ gaia), processed by the Gaia Data Processing and Analysis Consortium (DPAC, https://www.cosmos.esa.int/web/gaia/ dpac/consortium). Funding for the DPAC has been provided by national institutions, in particular the institutions participating in the Gaia Multilateral Agreement.
Facilities: HST (WFC3), Gemini (FLAMINGOS-2).

Software: DrizzlePac within PyRAF (Gonzaga et al. 2012), BUNDLE software (Anderson 2014), IRAF (Tody 1986), GAIA of the JAC Starlink Project (Draper et al. 2016).

\section{Appendix \\ PSF Subtraction on the FLT Images}

We used several reference sources to check and evaluate the performance of the PSF subtraction. Their locations in the WFC3/F160W image are indicated in Figure 5. Figures 2 and 6 show the corresponding images before and after the PSF subtraction. Aiming for a representation of all reference sources and the target in one plot, we obtained image statistics in the central $0.17 \times 0$.! 7 region of each bundlestack image for both, before and after the PSF subtraction. If a source is not well subtracted, one expects to see abnormally high mean and possibly abnormally high standard deviations in the sky/PSF-subtracted image in comparison to the reference sources. Since we consider a magnitude range (STmag from 19.0 to 28.7), we weigh the PSF-subtracted values with the respective ones from the image where the PSF was not subtracted. The resulting plot can be seen in Figure 7. The measures for the target clearly deviate (larger mean, larger standard deviation) from those of the other reference sources. 


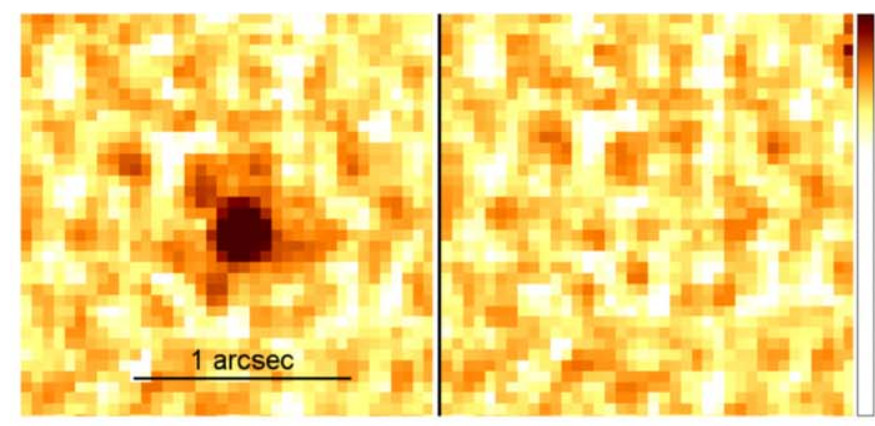

reference source $1, \mathrm{STmag}=27.3, \mathrm{R}_{\operatorname{maxmin}}=0.15$

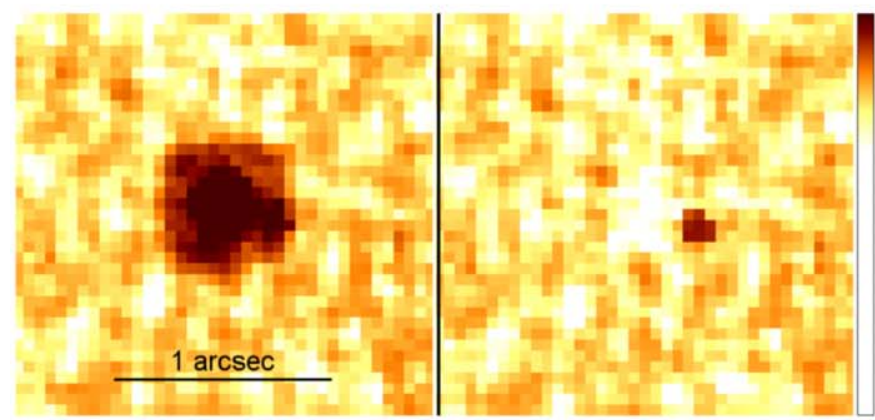

reference source $3, \mathrm{STmag}=26.5, \mathrm{R}_{\operatorname{maxmin}}=0.09$

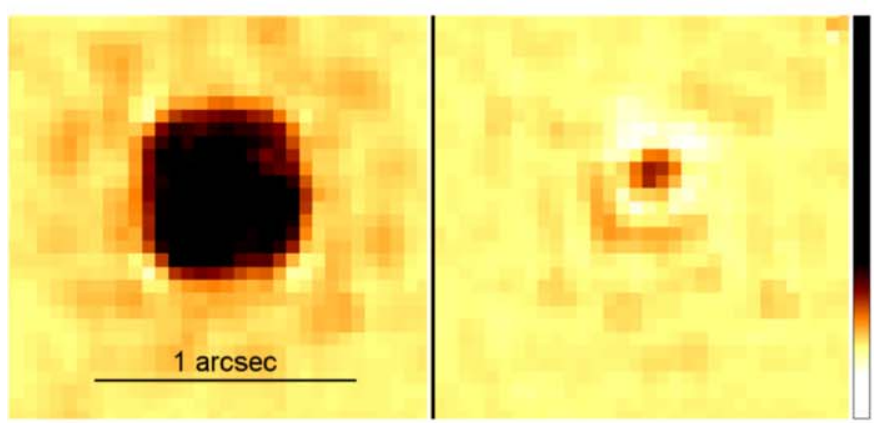

reference source 5, $\mathrm{STmag}=23.1, \mathrm{R}_{\operatorname{maxmin}}=0.03$
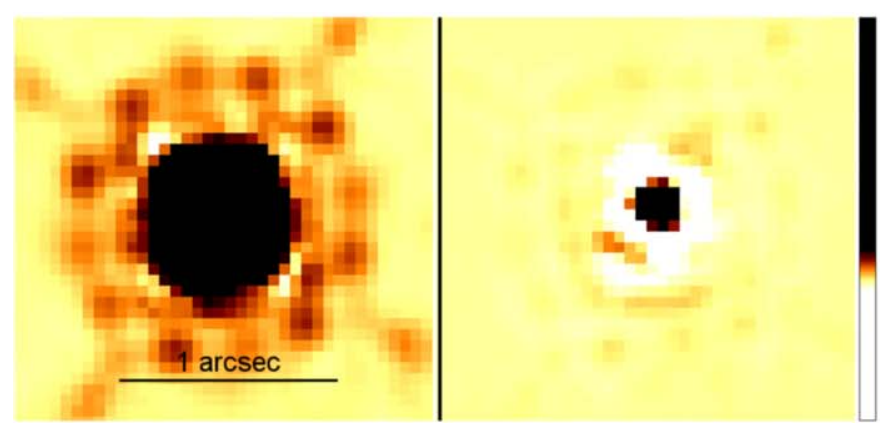

reference source $7, \mathrm{STmag}=19.0, \mathrm{R}_{\operatorname{maxmin}}=0.06$

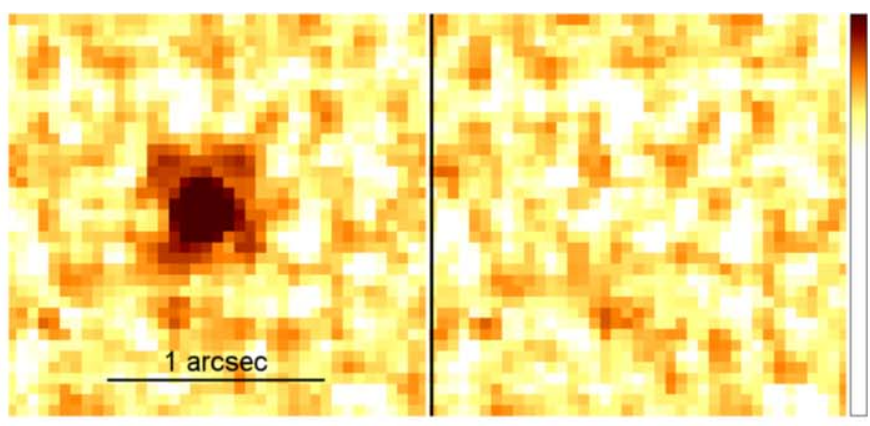

reference source $2, \mathrm{STmag}=27.1, \mathrm{R}_{\operatorname{maxmin}}=0.09$

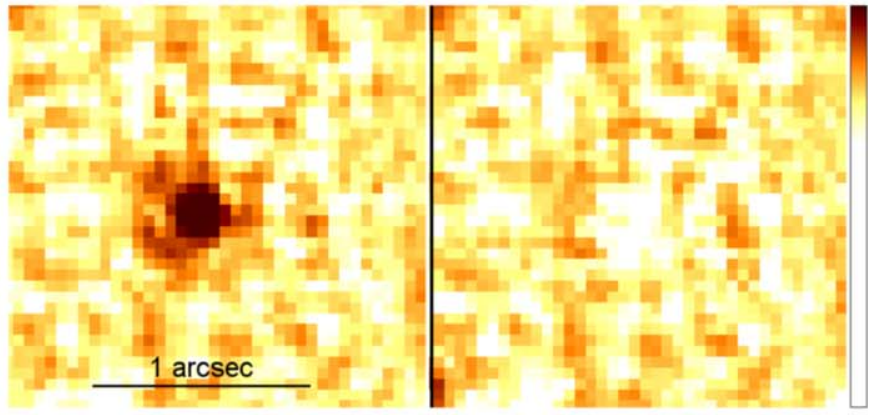

reference source $4, \mathrm{STmag}=27.5, \mathrm{R}_{\operatorname{maxmin}}=0.17$
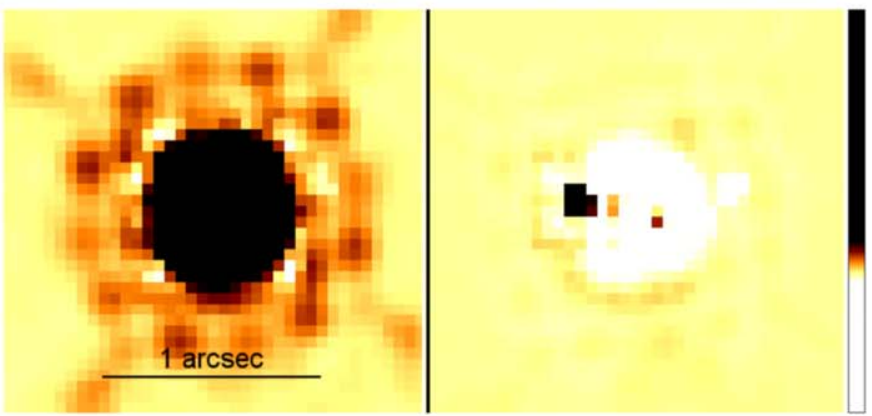

reference source $6, \mathrm{STmag}=19.1, \mathrm{R}_{\operatorname{maxmin}}=0.04$

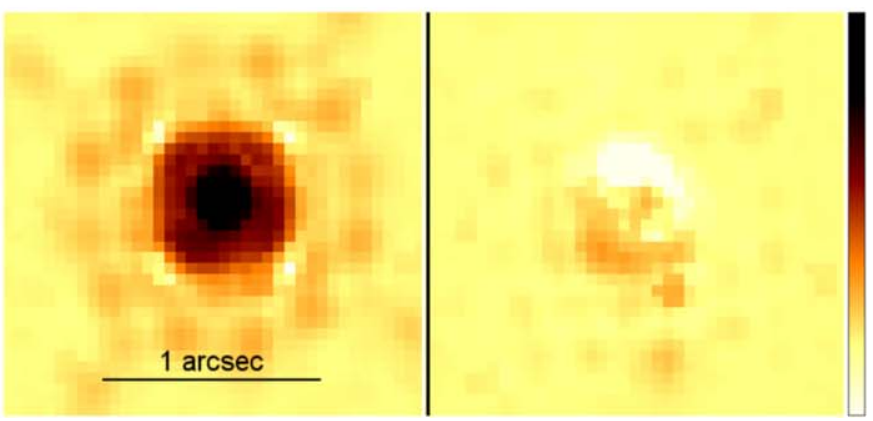

reference source $8, \mathrm{STmag}=21.5, \mathrm{R}_{\operatorname{maxmin}}=0.01$

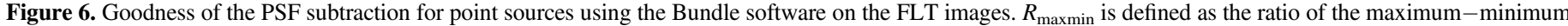

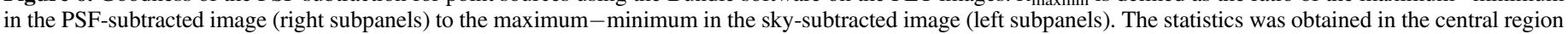

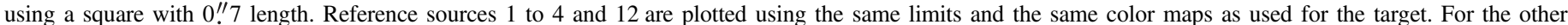
reference sources, we adapted the limits and color maps to better show the larger dynamical ranges. 


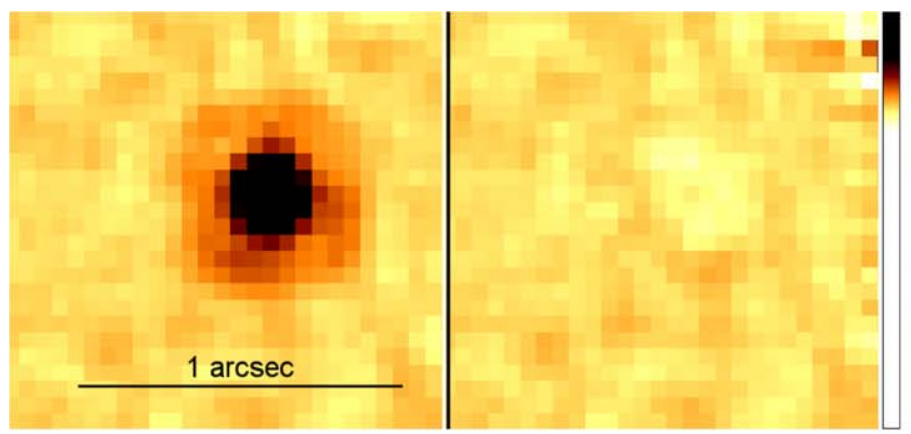

reference source $9, \mathrm{STmag}=26.5, \mathrm{R}_{\operatorname{maxmin}}=0.07$

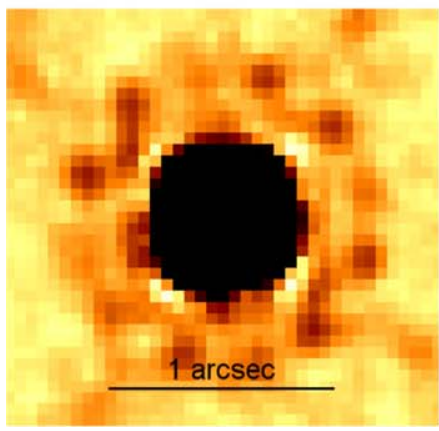

reference source $11, \mathrm{STmag}=22.6, \mathrm{R}_{\operatorname{maxmin}}=0.01$

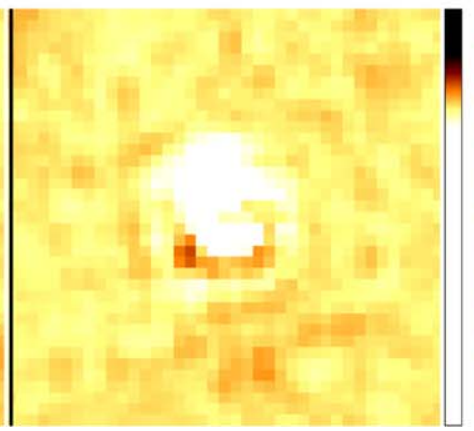

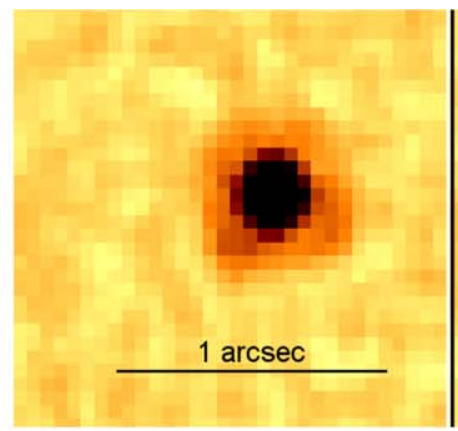

reference source $10, \mathrm{STmag}=26.5, \mathrm{R}_{\operatorname{maxmin}}=0.05$

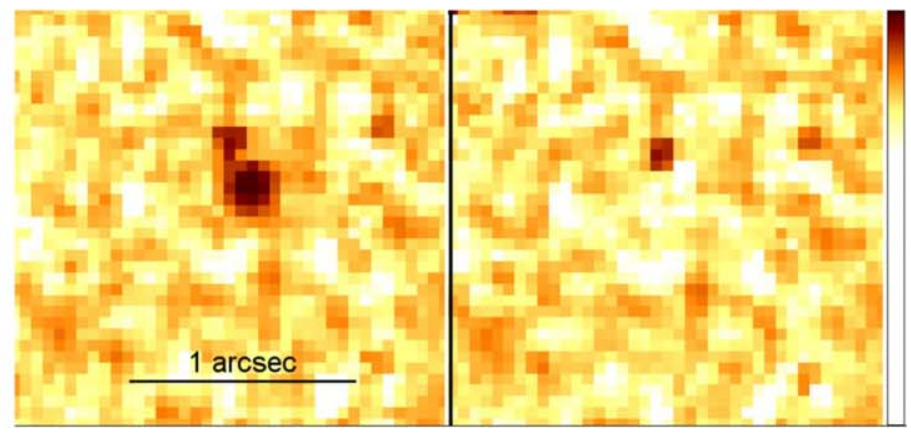

reference source $12, \mathrm{STmag}=28.7, \mathrm{R}_{\operatorname{maxmin}}=0.62$

Figure 6. (Continued.)

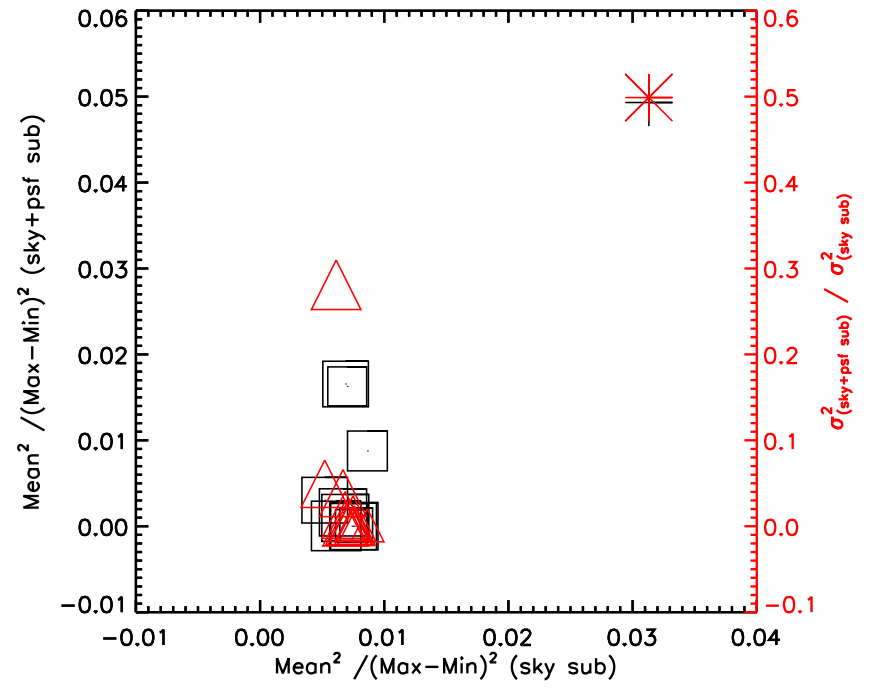

Figure 7. Comparison of image statistics in the inner $0.7 \times 0$. 7 regions for the (bundle-stack) images with PSF subtraction ("psf+sky sub") and without PSF subtraction ("sky sub") for the reference sources (boxes and triangles) and the target (cross and asterisk symbols). The sizes of the symbols scale with the F160W magnitude. The brightest reference source (smallest symbol) has STmag $=19.0$, and the faintest reference source has STmag $=28.7$. The latter is the object that is deviating from the other reference sources. Its PSFsubtracted image reveals a potential nearby faint neighbor.

\section{ORCID iDs}

B. Posselt (iD https://orcid.org/0000-0003-2317-9747

G. G. Pavlov (i) https://orcid.org/0000-0002-7481-5259

Ü. Ertan (1) https://orcid.org/0000-0002-1898-7902

K. L. Luhman (iD https://orcid.org/0000-0003-2822-2951

C. C. Williams (i) https://orcid.org/0000-0003-2919-7495

\section{References}

Anderson, J. 2014, Local Pixel Bundles: Bringing the Pixels to the People, Tech. Rep. ISR 2014-24, http://www.stsci.edu/hst/wfc3/documents/ISRs Anderson, J. 2016, Empirical Models for the WFC3/IR PSF, Tech. Rep. ISR 2016-12, http://www.stsci.edu/hst/wfc3/documents/ISRs

Anthonioz, F., Ménard, F., Pinte, C., et al. 2015, A\&A, 574, A41

Armitage, P. J. 2010, Astrophysics of Planet Formation (Cambridge: Cambridge Univ. Press)

Bertsch, D. L., Brazier, K. T. S., Fichtel, C. E., et al. 1992, Natur, 357, 306

Black, J. H., \& van Dishoeck, E. F. 1987, ApJ, 322, 412

Brownsberger, S., \& Romani, R. W. 2014, ApJ, 784, 154

Burgh, E. B., France, K., \& McCandliss, S. R. 2007, ApJ, 658, 446

Burrows, A., Hubbard, W. B., Lunine, J. I., \& Liebert, J. 2001, RvMP, 73, 719 Capitanio, L., Lallement, R., Vergely, J. L., Elyajouri, M., \& Monreal-Ibero, A. 2017, A\&A, 606, A65

Chiang, E. I., \& Goldreich, P. 1997, ApJ, 490, 368

Cutri, R. M., Skrutskie, M. F., van Dyk, S., et al. 2003, yCat, 2246

Daddi, E., Cimatti, A., \& Renzini, A. 2000, A\&A, 362, L45

Dame, T. M., Hartmann, D., \& Thaddeus, P. 2001, ApJ, 547, 792

Danilenko, A. A., Zyuzin, D. A., Shibanov, Y. A., \& Zharikov, S. V. 2011, MNRAS, 415, 867

Deller, A. T., Tingay, S. J., Bailes, M., \& Reynolds, J. E. 2009, ApJ, 701, 1243

Dobashi, K., Uehara, H., Kandori, R., et al. 2005, PASJ, 57, S1

Draine, B. T. 2003, ApJ, 598, 1017

Draine, B. T., \& Li, A. 2001, ApJ, 551, 807

Draper, P., Gray, N., Berry, D. S., \& Taylor, M. 2016, GAIA-Graphical Astronomy and Image Analysis Tool, http://star-www.dur.ac.uk/ pdraper/ gaia/gaia.html

Dullemond, C. P., \& Monnier, J. D. 2010, ARA\&A, 48, 205

Durant, M., \& van Kerkwijk, M. H. 2005, ApJ, 627, 376

Durant, M., van Kerkwijk, M. H., \& Hulleman, F. 2004, in IAU Symp. 218, Young Neutron Stars and Their Environments, ed. F. Camilo \& B. M. Gaensler (San Francisco, CA: ASP), 251

Eikenberry, S., Bandyopadhyay, R., Bennett, J. G., et al. 2012, Proc. SPIE, 8446, 84460

Ertan, Ü, Çalışkan, Ş, \& Alpar, M. A. 2017, MNRAS, 470, 1253

Ertan, Ü, Çalışkan, Ş, Benli, O., \& Alpar, M. A. 2014, MNRAS, 444, 1559

Ertan, Ü, Erkut, M. H., Ekşi, K. Y., \& Alpar, M. A. 2007, ApJ, 657, 441

Faherty, J., Walter, F. M., \& Anderson, J. 2007, Ap\&SS, 308, 225 
Foight, D. R., Güver, T., Özel, F., \& Slane, P. O. 2016, ApJ, 826, 66

Foster, J. B., \& Goodman, A. A. 2006, ApJL, 636, L105

Franx, M., Labbé, I., Rudnick, G., et al. 2003, ApJL, 587, L79

Fruchter, A. S., Hack, W., Dencheva, N., Droettboom, M., \& Greenfield, P. 2010, in 2010 Space Telescope Science Institute Calibration Workshop, ed. S. Deustua \& C. Oliveira (Baltimore, DC: STSci), 382

Gaia Collaboration, Brown, A. G. A., Vallenari, A., et al. 2016a, A\&A, 595, A2

Gaia Collaboration, Prusti, T., de Bruijne, J. H. J., et al. 2016b, A\&A, 595, A1

Giavalisco, M. 2002, ARA\&A, 40, 579

Golimowski, D. A., Leggett, S. K., Marley, M. S., et al. 2004, AJ, 127, 3516

Gonzaga, S., Hack, W., Fruchter, A., \& Mack, J. 2012, The DrizzlePac Handbook (Baltimore, MD: STSci)

Guo, Y., Giavalisco, M., Cassata, P., et al. 2012, ApJ, 749, 149

Haberl, F. 2007, Ap\&SS, 308, 181

Haberl, F. 2013, in The Fast and the Furious: Energetic Phenomena in Isolated Neutron Stars, Pulsar Wind Nebulae and Supernova Remnants, ed. J.-U. Wind (Paris: ESA), 6

Haberl, F., Motch, C., Zavlin, V. E., et al. 2004, A\&A, 424, 635

Hollenbach, D., \& McKee, C. F. 1989, ApJ, 342, 306

Kaplan, D. L., Kamble, A., van Kerkwijk, M. H., \& Ho, W. C. G. 2011, ApJ, 736, 117

Kaplan, D. L., \& van Kerkwijk, M. H. 2005, ApJL, 628, L45

Kaplan, D. L., \& van Kerkwijk, M. H. 2009, ApJ, 705, 798

Kargaltsev, O., \& Pavlov, G. G. 2008, in AIP Conf. Ser. 983, 40 Years of Pulsars: Millisecond Pulsars, Magnetars and More, ed. C. Bassa et al. (Melville, NY: AIP), 171

Kennel, C. F., \& Coroniti, F. V. 1984, ApJ, 283, 694

Kimble, R. A., MacKenty, J. W., O’Connell, R. W., \& Townsend, J. A. 2008, Proc. SPIE, 7010, 70101E

Lee, J.-J., Koo, B.-C., Lee, Y.-H., et al. 2014, MNRAS, 443, 2650

Lehtinen, K., \& Mattila, K. 1996, A\&A, 309, 570

Melis, C., Jura, M., Albert, L., Klein, B., \& Zuckerman, B. 2010, ApJ, 722, 1078

Mignani, R. P., De Luca, A., Hummel, W., et al. 2012, A\&A, 544, A100

Motch, C., Pires, A. M., Haberl, F., Schwope, A., \& Zavlin, V. E. 2009, A\&A, 497,423
Mulders, G. D., Min, M., Dominik, C., Debes, J. H., \& Schneider, G. 2013, A\&A, 549, A112

Posselt, B., \& Luhman, K. L. 2016, AN, 337, 576

Posselt, B., Neuhäuser, R., \& Haberl, F. 2009, A\&A, 496, 533

Posselt, B., Pavlov, G. G., Popov, S., \& Wachter, S. 2014, ApJS, 215, 3

Posselt, B., Popov, S. B., Haberl, F., et al. 2007, Ap\&SS, 308, 171

Rafelski, M., Teplitz, H. I., Gardner, J. P., et al. 2015, AJ, 150, 31

Rangelov, B., Pavlov, G. G., Kargaltsev, O., et al. 2017, ApJ, 835, 264

Reipurth, B., \& Bally, J. 2001, ARA\&A, 39, 403

Reynolds, S. P., Pavlov, G. G., Kargaltsev, O., et al. 2017, SSRv, 207, 175

Savage, B. D., Bohlin, R. C., Drake, J. F., \& Budich, W. 1977, ApJ, 216, 291

Sellgren, K. 1984, ApJ, 277, 623

Shibanov, Y. A., Koptsevich, A. B., Sollerman, J., \& Lundqvist, P. 2003, A\&A, 406, 645

Shibanov, Y. A., Zharikov, S. V., Komarova, V. N., et al. 2006, A\&A, 448,313

Shibuya, T., Ouchi, M., \& Harikane, Y. 2015, ApJS, 219, 15

Shull, J. M., \& Beckwith, S. 1982, ARA\&A, 20, 163

Skelton, R. E., Whitaker, K. E., Momcheva, I. G., et al. 2014, ApJS, 214, 24

Su, K. Y. L., Rieke, G. H., Malhotra, R., et al. 2013, ApJ, 763, 118

Tetzlaff, N., Eisenbeiss, T., Neuhäuser, R., \& Hohle, M. M. 2011, MNRAS, 417, 617

Tody, D. 1986, Proc. SPIE, 627, 733

van der Wel, A., Franx, M., van Dokkum, P. G., et al. 2014, ApJ, 788, 28

van Dokkum, P. G., Quadri, R., Marchesini, D., et al. 2006, ApJL, 638, L59

van Kerkwijk, M. H., \& Kaplan, D. L. 2008, ApJL, 673, L163

van Kerkwijk, M. H., \& Kulkarni, S. R. 2001, A\&A, 380, 221

Verbiest, J. P. W., Weisberg, J. M., Chael, A. A., Lee, K. J., \& Lorimer, D. R. 2012, ApJ, 755, 39

Viganò, D., Rea, N., Pons, J. A., et al. 2013, MNRAS, 434, 123

Voit, G. M. 1991, ApJ, 379, 122

Walter, F. M., Eisenbeiß, T., Lattimer, J. M., et al. 2010, ApJ, 724, 669

Wang, Z., Chakrabarty, D., \& Kaplan, D. L. 2006, Natur, 440, 772

Wang, Z., Kaplan, D. L., Slane, P., Morrell, N., \& Kaspi, V. M. 2013, ApJ, 769, 122

Williams, C. C., Curtis-Lake, E., Hainline, K. N., et al. 2018, ApJS, 236, 33

Zubko, V., Dwek, E., \& Arendt, R. G. 2004, ApJS, 152, 211 